\title{
A REMARK ON THE QUESTION OF UNIQUENESS FOR THE TRICOMI PROBLEM
}

\author{
L. M. SIBNER ${ }^{1}$
}

Uniqueness theorems for the Tricomi problem for the generalized Tricomi equation

$$
L u=K(y) u_{x x}+u_{y y}=0,
$$

where $K(y)$ is a monotone increasing function with $K(0)=0$, have been obtained under various restrictions either on the domain or on the function $K(y)$.

A theorem of Protter's (see [2]) states that uniqueness holds for any $K$ provided that the maximum vertical height of the elliptic domain is not too large. This vertical height depends on the particular $K$ chosen. However, for every smooth $K(y)$ there is a constant $y_{m}>0$, such that if the elliptic curve lies below $y=y_{m}$, then the Tricomi problem has a unique solution.

This result of Protter together with the fact that the Dirichlet problem for any uniformly elliptic equation has a unique solution under the most general conditions, leads to the conjecture that uniqueness holds without any unnatural restrictions on the function $K(y)$ or the shape of the elliptic domain.

The result below is not new (see, for example, [7] and [8]). Its interest lies in the fact that the method of proof is totally different from the usual uniqueness proofs for mixed problems which generally rely either upon energy integral methods or upon a maximum principle argument.

In the following, we consider the homogeneous equation (1) in the domain $D=D_{1} \cup D_{2}$ shown in Figure 1 , where $C_{1}$ and $C_{2}$ are characteristics of (1) and the elliptic curve $C_{0}$ includes the "normal curve" of Tricomi for any small distance at $A$ and $B$. We make no other restrictive assumptions about either $C_{0}$ or $K(y)$. For classical solutions, we shall prove the following

REMARK. There exist at most a finite number of linearly independent solutions of $L u=0$ which vanish on $C_{0}$ and $C_{1}$.

To prove this, let us assume that $u(x, y)$ is a nontrivial solution of this homogeneous problem for a given $K(y)$. Let $y=y_{m}$ be the appropriate maximum vertical height for this $K$. It follows that on some line, say $y=y_{0}<y_{m}, u\left(x, y_{0}\right)$ is not identically zero. Otherwise, it fol-

Received by the editors November 5, 1966.

${ }^{1}$ Research sponsored by N.S.F. Grant GP-4079 at Stanford University. 
lows from [2] that $u$ vanishes identically in $D$ for $y \leqq y_{m}$, and then from the maximum principle for elliptic equations, $u$ vanishes in all of $D$.

Let $u\left(x, y_{0}\right)=f(x)$ where $f$ is not identically zero. Denote by $D^{\prime}$ the intersection of $D$ with the half-plane: $y \leqq y_{0}$. Let $u^{\prime}(x, y)$ be the solution of (1) which vanishes on $C_{1}$ and on the part of $C_{0}$ which bounds $D^{\prime}$, but assumes the value $f(x)$ on the line $y=y_{0}$. By uniqueness, $u$ and $u^{\prime}$ agree in $D^{\prime}$.

Inside the elliptic part of $D^{\prime}$, the solution $u^{\prime}(x, y)$ may be represented as a certain integral operator on the function $f$ (see [4], [5], [6]). We give here a very brief description of how this representation may be obtained.

One can show that the solution $u^{\prime}(x, y)$ may be represented as the sum of a certain solution of the Tricomi equation, $U(x, y)$, and a solution, $V(x, y)$, of an inhomogeneous equation of the form (1). Then, making use of the fundamental solutions of the Tricomi equation, which are known, and of classical potential theoretic methods, the functions $U$ and $V$ may be represented as integral operators on the boundary values $f(x)$. In fact, on a line $y=y^{\prime}, 0<y^{\prime}<y_{0}$, we have

$$
u^{\prime}\left(x, y^{\prime}\right)=T f
$$

where Tf has Hölder continuous derivatives with any exponent $\beta$, $0<\beta<1$, for $f$ in some Hölder class, say, $C^{\alpha}$ (see [6]).

Let $D^{\prime \prime}$ be the intersection of $D$ with the half-plane: $y \geqq y^{\prime}$. Let $u^{\prime \prime}$ be the solution of (1) in $D^{\prime \prime}$ which vanishes on $C_{0}$ and is equal to Tf on the line: $y=y^{\prime}$. From elliptic theory, $u^{\prime \prime}(x, y) \in C^{1+\gamma}\left(D^{\prime \prime}\right)$, where $\gamma$ depends only on the shape of $D^{\prime \prime}$. Furthermore, $u^{\prime \prime}$ and $u$ agree on the boundary of $D^{\prime \prime}$. Hence, they agree in $D^{\prime \prime}$. It follows that $u^{\prime \prime}\left(x, y_{0}\right)$ $=f(x)$.

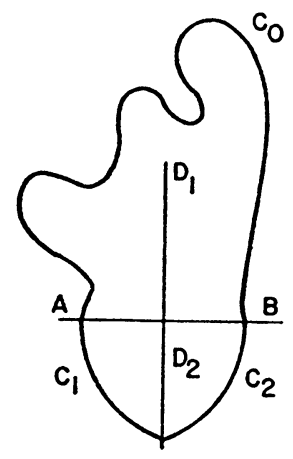

FIGURE 1 
Setting $S f=u^{\prime \prime}\left(x, y_{0}\right)$, we see that for $\alpha$ sufficiently small, $S$ is a compact linear operator on $C^{\alpha}$.

If one is not an eigenvalue of the operator $S$ then the relation

$$
S f=f
$$

would imply $f \equiv 0$.

At any rate, $S f=f$ can be satisfied by at most a finite number of linearly independent functions $f \in C^{\alpha}$.

The remark is also valid if the elliptic domain is unbounded provided, of course, that the solutions are assumed to vanish at infinity of the appropriate order.

I want to thank Professor M. H. Protter for his observation that the technique of overlapping domains (which I used in a different connection) could be applied to the uniqueness question.

\section{BIBLIOGRAPHY}

1. C. S. Morawetz, A uniqueness theorem for Frankl's problem, Comm. Pure Appl. Math. 7 (1954), 697-703.

2. M. H. Protter, Uniqueness theorems for the Tricomi problem, Part I, J. Rational Mech. Anal. 2 (1953), 107-114.

3. S. Agmon, L. Nirenberg and M. H. Protter, A maximum principle for a class of hyperbolic equations and applications to equations of mixed elliptic-hyperbolic type, Comm. Pure Appl. Math. 6 (1953), 455-470.

4. M. H. Protter, An existence theorem for the generalized Tricomi problem, Duke Math. J. 21 (1954), 1-8.

5. S. Gellerstedt, Sur un problème aux limites pour une équation linéaire aux derivées partielles $d u$ second ordre de type mixte, Thesis, Uppsala, 1935.

6. L. M. Sibner, A boundary value problem for an equation of mixed type having two transitions, J. Differential Equations (to appear).

7. C. S. Morawetz, Mixed equations and transonic flow, Rend. Math. e Appl. 25 (1966).

8. - Note on a maximum principle and a uniqueness theorem for an elliptichyperbolic equation, Proc. Roy. Soc. Ser. A 236 (1956), 141-144.

STANFORD UNIVERSITY 